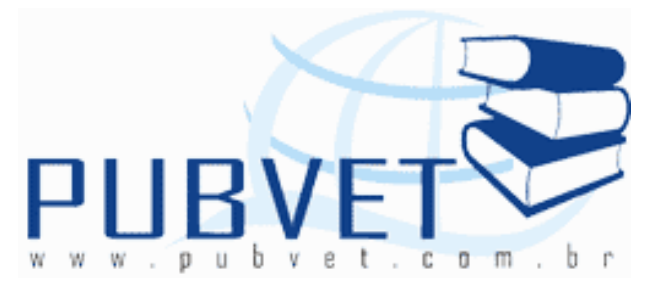

PUBVET, Publicações em Medicina Veterinária e Zootecnia.

\title{
Suprimento arterial para as glândulas adrenais de lobo marinho Arctocephalus australis Zimmerman, 1783 (Pinnipedia, Otariidae)
}

\author{
André Luiz Quagliatto Santos ${ }^{1}$, Lorena Tannús Menezes ${ }^{2}$, Fernando Moraes \\ Machado Brito ${ }^{3}$, Arthur Paulino Sanzo Kaminishi ${ }^{2}$, Tatiana Grillo Leonardo ${ }^{2}$, \\ Tatiana Lucena Pimentel ${ }^{5}$, Natália Siqueira D'Aparecida ${ }^{4}$
}

Laboratório de Ensino e Pesquisas em Animais Silvestres - LAPAS, FAMEV/UFU, e-mail: quagliatto@famev.ufu.br 1. Docente. 2. Mestrandos. 3. Doutorando. 4. Graduanda.

5. IBAMA

\section{Resumo}

Estudou-se em um Arctocephalus australis, fêmea, adulto, proveniente do CETAS (Centro de Resgate e Triagem de Animais/ IBAMA) do Estado de Santa Catarina, Brasil, a irrigação de suas glândulas adrenais, com propósito de avaliar aspectos sistemáticos relativos à origem, quantidade e distribuição territorial dos ramos arteriais encarregados da nutrição de seus parênquimas. Para tanto foi injetada uma solução corada de Neoprene Látex "450", via artéria aorta descendente torácica e em seguida fixado com solução aquosa de formol a $10 \%$ via veias jugulares externas e posteriormente dissecados os vasos em questão. Tal conduta mostrou-nos que: a glândula adrenal direita é irrigada pela artéria aorta descendente abdominal através de um ramo, através de um ramo da artéria celíaca, três ramos da quinta artéria lombar 
direita e de três ramos da artéria renal direita. A glândula adrenal esquerda é vascularizada por dois ramos colaterais procedentes da artéria aorta descendente abdominal, um colateral da artéria celíaca e outro da quarta artéria lombar esquerda, além de outros dois ramos da artéria renal esquerda e um da artéria ovárica esquerda. Suas dimensões são 26,00 mm e 17,20 mm de comprimento, $9,40 \mathrm{~mm}$ e $9,30 \mathrm{~mm}$ de largura e $3,15 \mathrm{~mm}$ e $7,00 \mathrm{~mm}$ de espessura, respectivamente para a direita e esquerda.

Palavras-chave: Arctocephalus australis, Artérias, Glândula adrenal, Anatomia.

\title{
Arterial Supply for to adrenal glands of the South American fur seal Arctocephalus australis Zimmerman, 1783 (Pinnipedia, Otariidae)
}

\begin{abstract}
An anatomical study has been carried out on the arterial branching system for South American fur seal (Arctocephalus australis Zimmerman, 1783) adrenal glands blood supply. A female adult South American fur seal were injected through the external jugular veins with a colored solution of Neoprene latex. Soon after this step they were maintained for fixation in a $10 \%$ aqueous solution of formalin, being dissected right after this procedure. The following observations were then carried out: the right adrenal gland is supplied by one branch of the descendent aorta artery and celiac artery, by three branches of $5^{\text {th }}$ right lumbar artery and three branches of right renal artery. The left adrenal gland is supplied by two branches from the descendent aorta artery, one branch of the celiac artery, and the $4^{\text {th }}$ left lumbar artery, two branches of the left renal artery and one branch of the ovaric artery. They dimensions are $26.00 \mathrm{~mm}$ and $17.20 \mathrm{~mm}$ of length, $9.40 \mathrm{~mm}$ and $9.30 \mathrm{~mm}$ of width and 3.15 $\mathrm{mm}$ and $7.00 \mathrm{~mm}$ of thickness, respectively for the right and left.
\end{abstract}

Keywords: Artocephalus australis, Arteries, Adrenal gland, Anatomy. 
SANTOS, A.L.Q. et al. Suprimento arterial para as glândulas adrenais de lobo marinho Arctocephalus australis Zimmerman, 1783 (Pinnipedia, Otariidae). PUBVET, Londrina, V. 5, N. 11, Ed. 158, Art. 1069, 2011.

\section{INTRODUÇÃO}

O termo Pinnipedia deriva do latim pinnipes e significa "pés em forma de nadadeiras", e essas espécies estão incluídas na ordem Carnivora (RUOPPOLO, 2006). Segundo Novak (1991), a ordem Pinnipedia é de mamíferos aquáticos que ocorrem ao longo das geleiras e litorais, principalmente nas regiões polares e temperadas dos oceanos e mares contigos do mundo e também em algumas áreas tropicais e certamente em águas interiores. São três famílias recentes, 18 gêneros e 34 espécies. A família Otariidae compreende sete gêneros recentes e 18 espécies. O gênero Arctocephalus apresenta oito espécies que variam consideravelmente de tamanho, desde o pequeno $A$. galapagoensis ao enorme A. pusillus. Em relação ao Arctocephalus australis, os machos adultos podem atingir até 189 centímetros de comprimento e seu peso varia entre $150-200 \mathrm{~kg}$ e as fêmeas adultas atingem 143 centímetros de comprimento e o peso entre $30-60 \mathrm{~kg}$.

Pinedo et al. (1992) citam que o A. australis também é conhecido como lobo marinho, lobo de dois pelos ou ainda "South American fur seal". É uma espécie sul-americana, distribuindo-se desde o sul do Peru até o Rio de Janeiro.

Anatomicamente, a adrenal consiste de duas unidades distintas: internamente a medula adrenal que tem origem neural e externamente o córtex adrenal, originado de células mesodérmicas, sendo essencial à vida, uma vez que seus hormônios principais influenciam o metabolismo de carboidratos, proteínas, lipídeos, eletrólitos e água (SCHMIDT-NIELSEN, 2002).

Em relação ao suprimento arterial, tratadistas como Schwarze e Schröder (1972), Sisson e Grossman (1975), Evans e Christensen (1979), Frandson (1979), Getty (1986) e Dyce et al. (1997), comentam de maneira superficial que as glândulas adrenais estão difusamente nutridas pelos ramos dos vasos adjacentes às artérias aorta, renal, frênico abdominal e lombares. Outros autores (MACHADO et al. 1999; 2002; SILVA et al.,1993 a,b,c; 2003) abordaram o suprimento arterial das glândulas adrenais em outras espécies como suínos, caprinos, roedores e lagomorfos, chegando a diversos resultados. 
SANTOS, A.L.Q. et al. Suprimento arterial para as glândulas adrenais de lobo marinho Arctocephalus australis Zimmerman, 1783 (Pinnipedia, Otariidae). PUBVET, Londrina, V. 5, N. 11, Ed. 158, Art. 1069, 2011.

Santos et al. (2004) estudaram em Arctocephalus australis o comportamento das artérias mesentéricas cranial e caudal, contudo, referências enfocando especificamente o suprimento arterial das glândulas adrenais nessa espécie, não foram encontradas. Entretanto, o tema é abordado na ordem carnívora, taxonomicamente mais próxima, através de trabalhos em cães (EVANS e CHRISTENSEN 1979), gatos S.R.D. (SEVERINO et al., 1992), gato mourisco (SANTOS et al. 2003) e gato tigrino (CARVALHO, et al., 2004).

O conhecimento da anatomia das glândulas se revela importante para subsidiar trabalhos como o de Yilmaz e Girgin (2005) que observaram a estrutura da glândula adrenal de Hytrix cristata através de microscopia de luz e eletrônica ou ainda, estudos como o de Noorafshan et al. (2005), que avaliaram a influência na estrutura da glândula adrenal das proles de ratas gestantes expostas ao estresse.

Para que ocorra o perfeito funcionamento da glândula adrenal é necessário que as vias por onde chegam os estímulos bem como onde ocorre a drenagem dos hormônios se apresentem eficientes, propõe-se conhecer a irrigação das glândulas adrenais de um Arctocephalus australis, tendo ainda como finalidade acrescentar conhecimentos indispensáveis ao desenvolvimento da anatomia comparada entre mamíferos.

\section{MATERIAL E MÉTODOS}

Para o estudo da irrigação das glândulas adrenais, utilizou-se uma exemplar fêmea adulta, de Arctocephalus australis doado após seu óbito pelo CETAS (Centro de Resgate e Triagem de Animais Silvestres/ IBAMA-SC, Brasil) para o Laboratório de Pesquisa em Animais Silvestres da Universidade Federal de Uberlândia, Minas Gerais, Brasil. Com auxílio de material cirúrgico, efetuouse uma incisão no nível do sexto espaço intercostal até a cavidade torácica. Procedeu-se à identificação e canalização da artéria aorta em seu trajeto prédiafragmático e, a partir daí, em seu sistema vascular arterial, foi injetada solução corada de Neoprene Látex "450" (Du Pont do Brasil - Indústria 
Química). Em seguida, a peça foi fixada em solução aquosa de formol a $10 \%$. Decorrido tempo suficiente para a fixação, a peça foi dissecada com auxílio de material cirúrgico convencional e lupa monocular (Wild 10x). Para abordagem das artérias que irrigam as glândulas adrenais, foi feita abertura na cavidade abdominal mediante duas incisões, sendo uma no sentido dorso ventral tangenciando a borda caudal do arco costal e outra sobre a linha alba, chegando até a face cranial da sínfise pelvina e ainda, no nível dos processos transversais das vértebras lombares.

\section{RESULTADOS}

Ao analisarem-se as disposições arteriais das glândulas adrenais em Arctocephalus australis, verificou-se que ambas localizam-se cranialmente aos rins e possuem uma forte assimetria, principalmente quanto à forma, onde a glândula direita se apresenta mais comprida e delgada em relação à esquerda (Figura 1).

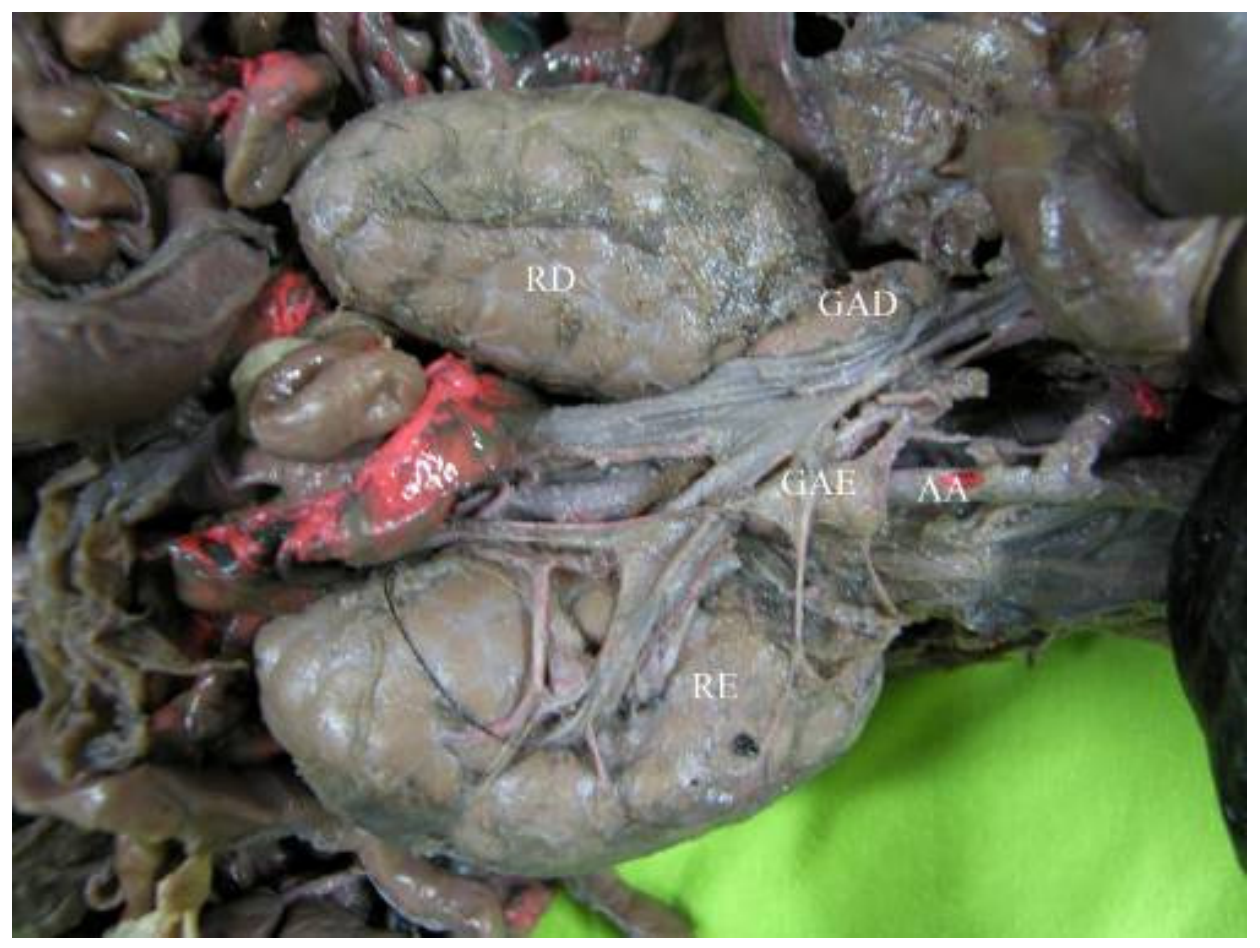

FIGURA 1. Fotografia das disposições arteriais das glândulas adrenais cranialmente aos rins de Arctocephalus australis. RD: Rim Direito; RE: Rim Esquerdo; GAD: Glândula Adrenal Direita; GAE: Glândula Adrenal Esquerda; AA: Artéria Aorta. 
SANTOS, A.L.Q. et al. Suprimento arterial para as glândulas adrenais de lobo marinho Arctocephalus australis Zimmerman, 1783 (Pinnipedia, Otariidae). PUBVET, Londrina, V. 5, N. 11, Ed. 158, Art. 1069, 2011.

A glândula adrenal direita é irrigada por um total de oito ramos, sendo um da artéria aorta descendente abdominal (12,50\%), um da celíaca $(12,50 \%)$, três ramos da quinta artéria lombar direita $(37,50 \%)$ e três ramos da artéria renal direita (37,50\%) (Tabela 1). Suas dimensões são 26,00 mm de comprimento, 9,40 $\mathrm{mm}$ de largura e 3,15 $\mathrm{mm}$ de espessura.

TABELA 1. Número de ramos das artérias que irrigam as glândulas adrenais de Arctocephalus australis. Uberlândia 2007.

\begin{tabular}{lll}
\hline Artérias/Glândula & $\begin{array}{l}\text { Adrenal } \\
\text { direita }\end{array}$ & $\begin{array}{l}\text { Adrenal } \\
\text { esquerda }\end{array}$ \\
\hline Aorta descendente & 1 & 2 \\
abdominal & & \\
Celíaca & 1 & 1 \\
Renal direita & 3 & 0 \\
Renal esquerda & 0 & 2 \\
Ovárica & 0 & 1 \\
Lombar direita & 3 & 0 \\
Lombar esquerda & 0 & 1 \\
\hline Total de ramos & 8 & 7 \\
\hline
\end{tabular}

A glândula adrenal esquerda é vascularizada por dois colaterais procedentes da artéria aorta descendente abdominal $(28,57 \%)$, um colateral da artéria celíaca $(14,29 \%)$, um ramo da quarta artéria lombar esquerda $(14,29 \%)$, dois colaterais da artéria renal esquerda $(28,57 \%)$ e ainda um colateral da artéria ovárica esquerda e outro da artéria lombar esquerda $(14,29 \%)$ (Tabela 1). Suas dimensões são 17,20 mm de comprimento, 9,30 $\mathrm{mm}$ de largura e 7,00 $\mathrm{mm}$ de espessura. 
SANTOS, A.L.Q. et al. Suprimento arterial para as glândulas adrenais de lobo marinho Arctocephalus australis Zimmerman, 1783 (Pinnipedia, Otariidae). PUBVET, Londrina, V. 5, N. 11, Ed. 158, Art. 1069, 2011.

\section{DISCUSSÃO}

Os achados, referentes à abordagem vascular arterial, relatados no Arctocephalus australis permitem fazer algumas considerações quando comparados àqueles referentes aos carnívoros encontrados na literatura. Severino et al. (1992) relatam que em gatos S.R.D. a irrigação pode ocorrer através de 1 a 4 ramos da artéria aorta abdominal, 1 a 3 ramos da artéria abdominal cranial, 1 ou 2 ramos da artéria renal esquerda, 1 ramo da artéria frênica caudal e dois ramos da artéria frênico abdominal. As variações encontradas para as artérias aorta abdominal e renal esquerda coincidem com as descritas neste trabalho, porém, o mesmo não ocorreu com as demais, pois os ramos não foram demonstrados. Em se tratando de literatura específica a respeito da irrigação das glândulas adrenais, a glândula adrenal direita pode ser irrigada por ramos da artéria abdominal cranial que variam entre um e três, por um único ramo da artéria renal direita, por ramos da artéria aorta abdominal, que variam entre um e três ramos, e por um ramo das artérias frênico abdominal, frênica caudal e lombar, cada. Estes resultados convergem com os encontrados neste trabalho, em relação aos ramos das artérias aorta descendente abdominal e lombar. No entanto, o número de ramos da artéria renal direita encontrado foi maior no Arctocephalus australis. Os ramos das demais artérias não foram descritos neste estudo.

Em um estudo relacionado a gato mourisco, Santos et al. (2003) concluíram que as glândulas adrenais nessa espécie recebem ramos das artérias aorta abdominal, renais direita e esquerda, o que coincide em parte com o relatado em Arctocephalus australis, mas também existem algumas diferenças no suprimento arterial das glândulas adrenais entre as duas espécies.

De acordo com Carvalho et al. (2004), a glândula adrenal direita de Leopardus trigrinus é vascularizada por quatro ramos provenientes diretamente da artéria frênica caudal, enquanto a da glândula adrenal esquerda ocorre por cinco ramos da artéria frênica caudal esquerda, um ramo da artéria aorta abdominal, três ramos da renal esquerda e dois ramos da 
SANTOS, A.L.Q. et al. Suprimento arterial para as glândulas adrenais de lobo marinho Arctocephalus australis Zimmerman, 1783 (Pinnipedia, Otariidae). PUBVET, Londrina, V. 5, N. 11, Ed. 158, Art. 1069, 2011.

artéria mesentérica cranial, o que difere bastante do ocorrido no presente estudo, tanto no que refere ao número total de vasos quanto às suas origens para ambas as glândulas.

\section{CONCLUSÕES}

As glândulas adrenais direita e esquerda do Arctocephalus australis recebem ramos das artérias aorta abdominal, celíaca, renal e lombar direitas, enquanto que a glândula adrenal esquerda recebe ramos das artérias aorta abdominal, celíaca, renal, lombar e ovárica esquerdas. Suas dimensões são $26,00 \mathrm{~mm}$ e $17,20 \mathrm{~mm}$ de comprimento, 9,40 $\mathrm{mm}$ e 9,30 $\mathrm{mm}$ de largura e $3,15 \mathrm{~mm}$ e $7,00 \mathrm{~mm}$ de espessura, respectivamente, para as glândulas direita e esquerda.

\section{AGRADECIMENTOS}

Os autores agradecem a José Truda Palazzo Junior, presidente de INC. BR. e a Jordan Wallauer, veterinário responsável, por terem concedido o material utilizado neste trabalho.

\section{REFERÊNCIAS}

CARVALHO, S.F.M.; SANTOS, A.L.Q.; PEREIRA, P.C.; MIRANDA, R.L.; GUIMARÃES, G.C.; ALVES JUNIOR, J.R.F.; DIAS, E.A.; PEREIRA, R.V.V.; MORAES, F.M.; ANDRADE, M.B.; BORGES, K.M. Forma, dimensões e irrigação arterial das glândulas adrenais do gato tigrino (Leopardus tigrinus - Gray, 1842) (Felidae). In: ENCONTRO CIENTÍFICO DE PÓS GRADUANDOS DA FCAV E ENCONTRO CIENTÍFICO DE PÓS - GRADUANDOS EM CIÊNCIAS AGROPECUÁRIAS DA UNESP, 4., 3., 2004, Jaboticabal. Anais... Jaboticabal: Universidade Estadual Paulista, 2004.

DYCE, K.M.; SACK W.O.; WENSING, C.J.G. Tratado de Anatomia Veterinária. 2.ed. Rio de Janeiro: Guanabara Koogan, 1997.

EVANS, H.E.; CHRISTENSEN, G.C. Miller's Anatomy of the Dog. 2. ed. Philadelphia: Sauders Company, 1979. 1181p.

FRANDSON, R.D. Anatomia e Fisiologia dos Animais Domésticos. 2. ed. Rio de Janeiro: Guanabara Koogan, 1979. 429p.

NOORAFSHAN, A; KARIMIPOOR, M; BAHMANPOOR, S.; DEHGHANI, $F$. The influence of exposure to stress of pregnant rats on the adrenal gland structure of their offspring. An unbiased stereological study. Scandinavian Journal of Laboratory Animal Science. Estocolmo, v.32, n.3, p.161-166, sept. 2005. 
SANTOS, A.L.Q. et al. Suprimento arterial para as glândulas adrenais de lobo marinho Arctocephalus australis Zimmerman, 1783 (Pinnipedia, Otariidae). PUBVET, Londrina, V. 5, N. 11, Ed. 158, Art. 1069, 2011.

MACHADO, G.V.; ROMAGNOLLI, P.; SOUZA, J.R.; TURQUETI, V.S.; ULIANA, S.M.; SILVA, M.H. Suprimento arterial para as glândulas adrenais no Ratão-do-banhado (Myocastor coypus Molina, 1782). Archives of Veterinary Science, Curitiba, v.7, n.2, p.9-14, dez. 2002.

MACHADO , G. V.; SILVA , F. O C.; MIGLINO , M. A ; SANTOS , T. C.; ANDRIOLI , L. G. Suprimento arterial para as glândulas adrenais em Lepus europaeus. Arquivo de ciências veterinárias e zoologia da UNIPAR, Umuarama, v.2, n.1, p.23-27, jun. 1999.

NOWAK, R.M. Walkes's mammals of the world. 5 ed. Baltimore: J.H. University Press, v.2, 1991. 1629p.

PINEDO, M.C.; ROSAS, F.C.W.; MARMONTEL, M. Cetáceos e pinípedes do Brasil: Uma revisão dos registros e guia para identificação das espécies. Manaus: United Nations Environmental Programme. (UNEP)/FUA, 1992.

RUOPPOLO, V. Pinnipedia (Lobo-marinho, Leão-marinho, Foca, Morsa). In: CUBAS, Z.S.; SILVA, J.C.R.; CATÃO-DIAS, J.L. Tratado de animais selvagens. São Paulo: Roca, 2006, cap 40 , p. $682-700$.

SANTOS, A. L. Q. ; CARVALHO, S. F. M. ; MORAES, F. M. ; MAGALHAES, L. M. ; ANDRADE, M. B. ; FERNANDES, S. M. L. ; BOSSO, A. C. S. . Forma, Dimensões e Irrigação Arterial das Glândulas Adrenais do Gato Mourisco (Herpailurus yagouaroundi- Svertzow, 1858) (FELIDAE). In: II Encontro Sobre Animais Selvagens, 2003, Poços de Caldas. Anais... , 2003. 1 CD-ROM.

SANTOS, A.L.Q.; MORAES, F.M.; CARVALHO, S.F.M.; ALVES JÚNIOR, J.R.F.; BORGES, K.M.; DIAS, E.A.; ANDRADE, M.B.; MARQUES, F.K.; LIMA, E.M.M. Comportamento das artérias mesentéricas cranial e caudal em Arctocephalus australis (Pinnipedia, Otariidae). Ars veterinária. Jaboticabal, v.20, n.3, p. 242-248, set. 2004.

SCHMIDT-NIELSEN, K. Controle hormonal. In: Fisiologia animal. 5 ed. São Paulo: Livraria Santos, 2002. cap 12, p. 497-531

SEVERINO, R.S.; SILVA, F.O.C.; SANTOS, A.L.Q.; DRUMMOND, S.S.; SILVA, M.; ESTEVÃO, E.R.M. Irrigação das glândulas adrenais em gatos sem raça definida. In: $X$ Semana Científica de Medicina Veterinária da Universidade Federal de Uberlândia, 10, 1992, Uberlândia. Anais... 1992, v.1, n.1, p.46-47.

SILVA, F.O.C.; SEVERINO, R.S.; SANTOS, A.L.Q.; DRUMMOND, S.S.; BOMBONATO, P.P.; BORGES, M. Suprimento arterial das glândulas adrenais em suínos da raça Large White. XI Semana Científica de Medicina Veterinária da Universidade Federal de Uberlândia. Anais..., 1993a, v.2, n. 1, p. 36.

SILVA, F.O.C.; SEVERINO, R.S.; SANTOS, A.L.Q.; DRUMMOND, S.S.; BOMBONATO, P.P.; GARGALHONE, A.G.; BRITO, L.F.C. Suprimento arterial das glândulas adrenais em fetos de suínos da raça Landrace. XI Semana Científica de Medicina Veterinária da Universidade Federal de Uberlândia, 1993. Anais..., 1993b, v.2, n. 1, p. 37.

SILVA, F.O.C.; SEVERINO, R.S.; SANTOS, A.L.Q.; DRUMMOND, S.S.; BOMBONATO, P.P.; BORGES, M. Suprimento arterial das glândulas adrenais em suínos sem raça definida. In: XI Semana Científica de Medicina Veterinária da Universidade Federal de Uberlândia, 11, 1993, Uberlândia. Anais..., 1993c, v.2, n. 1, p.38.

SILVA, F.O.C.; SEVERINO, R.S.; DRUMMOND, S.S.; MACHADO, G.V.; NOLASCO, R.M.; LIMA, E.M.M. Suprimento arterial para as glândulas adrenais em caprinos (Capra hircus) da raça Saanen. Arquivo de ciências veterinárias e zoologia da UNIPAR, Umuarama, v. 6, n.1, p. 9-14, jun. 2003.

SISSON, S.; GROSSMAN, J.D. Anatomia de los animals domesticos. 4 ed. Barcelona: Salvat, 1975, 952p. 
SANTOS, A.L.Q. et al. Suprimento arterial para as glândulas adrenais de lobo marinho Arctocephalus australis Zimmerman, 1783 (Pinnipedia, Otariidae). PUBVET, Londrina, V. 5, N. 11, Ed. 158, Art. 1069, 2011.

SISSON/GRossman. GeTtY, R. Anatomia dos Animais Domésticos. 6ed. Rio de Janeiro: Guanabara, 1986. v.6

SCHWARZE, E.; SCHRÖDER, L. Compêndio de Anatomia Veterinária. Zaragoza: Acribia, 1972. v. 4. 206p.

YILMAZ, S; GIRGIN, A. Light and electron microscopic observations on the structure of the porcupine (Hystrix cristata) adrenal gland. Veterinarski Arhiv, Zagreb, v. 75, n.3, p. 265272, june. 2005 